TRANSACTIONS OF THE

AMERICAN MATHEMATICAL SOCIETY

Volume 354, Number 9, Pages 3621-3637

S 0002-9947(02)03034- 9

Article electronically published on April 23, 2002

\title{
GLOBAL EXISTENCE AND NONEXISTENCE FOR NONLINEAR WAVE EQUATIONS WITH DAMPING AND SOURCE TERMS
}

\author{
MOHAMMAD A. RAMMAHA AND THERESA A. STREI
}

\begin{abstract}
We consider an initial-boundary value problem for a nonlinear wave equation in one space dimension. The nonlinearity features the damping term $|u|^{m-1} u_{t}$ and a source term of the form $|u|^{p-1} u$, with $m, p>1$. We show that whenever $m \geq p$, then local weak solutions are global. On the other hand, we prove that whenever $p>m$ and the initial energy is negative, then local weak solutions cannot be global, regardless of the size of the initial data.
\end{abstract}

\section{INTRODUCTION}

In this article, we study the initial-boundary value problem

$$
\begin{gathered}
u_{t t}-u_{x x}+|u|^{m-1} u_{t}=|u|^{p-1} u, \quad \text { in }(0,1) \times(0, T), \\
u(x, 0)=u^{0}(x), \quad u_{t}(x, 0)=u^{1}(x), \quad \text { in }(0,1), \\
u(0, t)=0, \quad u_{x}(1, t)=h(t), \quad \text { in }(0, T),
\end{gathered}
$$

where $m, p>1$ and $h \in C^{1}[0, \infty)$.

The evolution equation in (1.1) is a simple prototype of the more general equation

$$
u_{t t}-\Delta u+Q\left(x, t, u, u_{t}\right)=\mathcal{F}(x, u),
$$

where $Q$ and $\mathcal{F}$ satisfy the following structural conditions: $v Q(x, t, u, v) \geq 0$, $Q(x, t, u, 0)=\mathcal{F}(x, 0)=0$, and $\mathcal{F}(x, u) \sim|u|^{p-1} u$ for large $|u|$. Various examples of the general evolution equation above arise in physics. For instance, if $Q \equiv 0$ and $\mathcal{F}(x, u)=u^{3}$, or more generally, any positive odd power of $u$, then the equation arises in quantum field theory (cf. Jörgens [10] and Segal [23]). On the other hand, if $\mathcal{F} \equiv 0$ and $Q\left(x, t, u, u_{t}\right)=\left|u_{t}\right| u_{t}$, then the equation provides a model for a classical vibrating membrane with a resistance force that is proportional to the velocity $u_{t}$.

In this paper, we analyze the global well posedness of the initial-boundary value problem (1.1). It is well known [6, 15, 21] 25] that when the damping term $|u|^{m-1} u_{t}$ is absent from the equation, then the source term $|u|^{p-1} u$ drives the solution to (1.1) to blow up in finite time. However, the interaction between the damping

Received by the editors May 25, 2001.

2000 Mathematics Subject Classification. Primary 35L05, 35L20; Secondary 58K55.

Key words and phrases. Wave equations, weak solutions, blow-up.

The second author was supported in part by the National Physical Science Consortium and the National Security Agency. 
and source terms is often difficult to analyze. For example, we refer the reader to [5, 16, 18, 16] and the references therein.

There has been an extensive body of work on the global regularity of solutions to various hyperbolic equations. One of the pioneering papers in the area was by Lions and Strauss [20. We also note here the work of Georgiev and Todorova [5] and Levine and Serrin [16]. In [5] the authors analyzed the global regularity of solutions to a similar equation, but with a damping term of the form $\left|u_{t}\right|^{m-1} u_{t}$. However, the blow-up result obtained in [5] is for large data. In [16], Levine and Serrin proved several abstract theorems on the global nonexistence of solutions to a large class of nonlinear hyperbolic equations. Their results are applicable to the initial-boundary value problem (1.1) and they yield that local solutions to (1.1) cannot be global, whenever $p>m$ and the initial energy is negative. We remark here that our blow-up result in section 5 is not a verification of the abstract theorem in [16. Moreover, one can deduce from the work carried out in section 5 a precise upper bound for the life span of weak solutions to (1.1).

Due to the fact that the nonlinearity in the equation is not sufficiently regular, a standard fixed-point argument to establish the existence of weak solutions to (1.1) is not applicable. In section 3, we construct a local weak solution to (1.1) by utilizing an appropriate compactness theorem for the convergence of the Galerkin scheme in the appropriate spaces. In section 4 , we prove that the every local weak solution to (1.1) is global, provided $m \geq p$. The latter is achieved by establishing an energy-type estimate for the sequence of the approximate solutions. In section 5 , we use an argument similar to the one in [5] to prove that every local weak solution to (1.1) with negative initial energy blows up in finite time, regardless of the size of the initial data.

\section{Preliminaries}

In this section we introduce some notation, definitions, and the technical assumptions that are necessary for the remaining sections of the paper. Let $L^{2}(0,1)$ denote the standard Lebesgue space and $H^{s}(0,1)$ the standard Sobolev space. For $s>1 / 2$ we let

$$
H_{0,0}^{s}=H_{0,0}^{s}(0,1)=\left\{u \in H^{s}(0,1): u(0)=0\right\} .
$$

We introduce the operator: $A: L^{2}(0,1) \rightarrow L^{2}(0,1)$, where $A=-\frac{\partial^{2}}{\partial x^{2}}$ with its domain

$$
\mathcal{D}(A)=\left\{u \in H^{2}(0,1): u(0)=0, u^{\prime}(1)=0\right\} .
$$

It is well known that $A$ is positive and self-adjoint and is the inverse of a compact operator. Moreover, $A$ has the infinite sequence of positive eigenvalues $\left\{\lambda_{n}=\right.$ $\left.\left(\frac{(2 n-1) \pi}{2}\right)^{2}: n=1,2, \ldots\right\}$ and a corresponding sequence of eigenfunctions $\left\{e_{n}=\right.$ $\left.\sqrt{2} \sin \left(\lambda_{n}^{1 / 2} x\right): n=1,2, \ldots\right\}$ that forms an orthonormal basis for $L^{2}(0,1)$. Namely, if $u \in L^{2}(0,1)$, then $u=\sum_{n=1}^{\infty} u_{n} e_{n}$, where the convergence is in $L^{2}(0,1)$, with $\|u\|_{L^{2}(0,1)}^{2}=\sum_{n=1}^{\infty}\left|u_{n}\right|^{2}$ and $u_{n}=\left\langle u, e_{n}\right\rangle_{L^{2}(0,1)}$.

Let $X$ and $Y$ be Banach spaces. We write $X \hookrightarrow Y$ if $X$ is continuously imbedded in $Y$. More precisely, the natural injection $i: X \rightarrow Y$ is continuous, and we identify $X$ with the subspace $i(X)$ of $Y$. We denote by $\mathcal{L}(X, Y)$ the space of all continuous linear operators from $X$ to $Y$. 
The powers of $A$ are defined as follows:

$A^{s}: \mathcal{D}\left(A^{s}\right) \subseteq L^{2}(0,1) \rightarrow L^{2}(0,1), A^{s} u=\sum_{n=1}^{\infty} \lambda_{n}^{s} u_{n} e_{n}$, with the domain of $A^{s}$ given by

$$
\mathcal{D}\left(A^{s}\right)=\left\{u \in L^{2}(0,1): u=\sum_{n=1}^{\infty} u_{n} e_{n}, \sum_{n=1}^{\infty} \lambda_{n}^{2 s}\left|u_{n}\right|^{2}<\infty\right\} .
$$

We remark here that the results of Grisvard $[8$ and Seeley 22 give the following characterization for the fractional powers of $A$ :

$$
\mathcal{D}\left(A^{s}\right)=\left\{\begin{array}{l}
H^{2 s}(0,1), 0 \leq s<\frac{1}{4}, \\
H_{0,0}^{2 s}, \frac{1}{4}<s<\frac{3}{4}, \\
\left\{u \in H^{2 s}(0,1): u(0)=0, u^{\prime}(0)=0\right\}, \frac{3}{4}<s \leq 1 .
\end{array}\right.
$$

Moreover, $\mathcal{D}\left(A^{1 / 4}\right) \hookrightarrow H^{1 / 2}(0,1), \mathcal{D}\left(A^{3 / 4}\right) \hookrightarrow H_{0,0}^{3 / 2}$, and the norm $\|u\|_{H^{s}(0,1)}$ is equivalent to $\left(\sum_{n=1}^{\infty} \lambda_{n}^{s}\left|u_{n}\right|^{2}\right)^{1 / 2}$. Therefore, we set

$$
\|u\|_{H^{s}(0,1)}^{2}=\sum_{n=1}^{\infty} \lambda_{n}^{s}\left|u_{n}\right|^{2} .
$$

We also introduce $S(t)$ and $C(t)$, the sine and cosine operators associated with A. $S(t), C(t): L^{2}(0,1) \rightarrow L^{2}(0,1)$ are given by $S(t)=A^{-1 / 2} \sin \left(A^{1 / 2} t\right)$ and $C(t)=\cos \left(A^{1 / 2} t\right)$. More specifically, if $u \in L^{2}(0,1)$ with $u=\sum_{n=1}^{\infty} u_{n} e_{n}$, then

$$
S(t) u=\sum_{n=1}^{\infty} \lambda_{n}^{-1 / 2} \sin \left(\lambda_{n}^{1 / 2} t\right) u_{n} e_{n}, \quad C(t) u=\sum_{n=1}^{\infty} \cos \left(\lambda_{n}^{1 / 2} t\right) u_{n} e_{n} .
$$

The following assumptions will be valid throughout the paper:

(H1): $u^{0} \in H_{0,0}^{1}, u^{1} \in L^{2}(0,1)$.

(H2): $h \in C^{1}[0, \infty)$.

Also, throughout we set

$$
G(u)=\int_{0}^{u}|\xi|^{m-1} d \xi \quad \text { and } \quad f(u)=|u|^{p-1} u .
$$

The following lemma will be needed.

Lemma 2.1. The mappings $f$ and $G$ are continuous from $H_{0,0}^{1}$ into $H_{0,0}^{1}$.

Proof. Since the two functions are virtually identical, we present the proof for $G$. Let $v_{n} \in H_{0,0}^{1}$, be such that $v_{n} \rightarrow v$ in $H_{0,0}^{1}$. Since $H_{0,0}^{1} \hookrightarrow C^{0}[0,1]$ with

$$
\sup _{x \in[0,1]}\left|v_{n}(x)-v(x)\right| \leq\left\|v_{n}-v\right\|_{H^{1}}
$$

then $v_{n} \rightarrow v$ uniformly on $[0,1]$. So there exists a constant $C_{0}>0$ such that for all $n \geq 1$

$$
\sup _{x \in[0,1]}\left|v_{n}(x)\right|, \sup _{x \in[0,1]}|v(x)| \leq C_{0} .
$$

It follows from the mean value theorem that

$$
\left|G\left(v_{n}\right)-G(v)\right|=\left.\left|\int_{v}^{v_{n}}\right| \xi\right|^{m-1} d \xi \mid \leq C\left\|v_{n}-v\right\|_{\infty}
$$


for some constant $C>0$. Therefore, $G\left(v_{n}\right) \rightarrow G(v)$ uniformly on [0,1]. In particular, $G\left(v_{n}\right) \rightarrow G(v)$ in $L^{2}(0,1)$. Now,

$$
\begin{aligned}
& \left\|\partial_{x}\left(G\left(v_{n}\right)-G(v)\right)\right\|_{L^{2}}=\left\|\left|v_{n}\right|^{m-1} \partial_{x} v_{n}-|v|^{m-1} \partial_{x} v\right\|_{L^{2}} \\
& \quad \leq\left\|\left|v_{n}\right|^{m-1}\left(\partial_{x} v_{n}-\partial_{x} v\right)\right\|_{L^{2}}+\| \partial_{x} v\left(\left|v_{n}\right|^{m-1}-|v|^{m-1} \|_{L^{2}}\right. \\
& \leq C\left\|v_{n}-v\right\|_{H^{1}}+\|v\|_{H^{1}}\left\|\left|v_{n}\right|^{m-1}-|v|^{m-1}\right\|_{L^{2}} \rightarrow 0,
\end{aligned}
$$

since $v_{n} \rightarrow v$ in $H^{1}(0,1)$ and $\left|v_{n}\right|^{m-1} \rightarrow|v|^{m-1}$ in $L^{2}(0,1)$.

Hence, $\left\|G\left(v_{n}\right)-G(v)\right\|_{H^{1}} \rightarrow 0$ as $n \rightarrow \infty$. The fact that $\left.G\left(v_{n}\right)\right|_{x=0}=\left.G(v)\right|_{x=0}$ $=0$ is trivial.

Due to the lack of smoothness of the nonlinearity in the equation, one could anticipate that the solution of the initial-boundary value problem (1.1) would not be highly regular. Therefore, we shall use the weak formulation of the problem to define a solution.

Definition 2.2. Let $u^{0} \in H_{0,0}^{1}, u^{1} \in L^{2}(0,1)$. We say that $u$ is a weak solution to the initial-boundary value problem $(1.1)$ on $[0, T]$ if $u \in L^{2}\left(0, T, H_{0,0}^{1}\right), u^{\prime} \in$ $L^{2}\left(0, T, L^{2}(0,1)\right)$ and $u$ satisfies

$$
\begin{gathered}
\left\langle u^{\prime}(t), \phi\right\rangle_{L^{2}(0,1)}-\left\langle u^{1}, \phi\right\rangle_{L^{2}(0,1)}+\int_{0}^{t}\left\langle A^{1 / 2} u(s), A^{1 / 2} \phi\right\rangle_{L^{2}(0,1)} d s \\
-\int_{0}^{t}\left[h(s) \phi(1)+\langle f(u(s)), \phi\rangle_{L^{2}(0,1)}\right] d s \\
+\langle G(u(t)), \phi\rangle_{L^{2}(0,1)}-\left\langle G\left(u^{0}\right), \phi\right\rangle_{L^{2}(0,1)}=0
\end{gathered}
$$

for all $\phi \in H_{0,0}^{1}$ and almost every $t \in[0, T]$.

In order for us to easily obtain certain estimates, we now derive the integral equations that must be satisfied by a weak solution to the initial-boundary value problem (1.1). Let $v(t)=u(t)-w(t)$, where $w(t)=x h(t)$. Then, $v$ formally satisfies the following abstract initial-value problem:

$$
\begin{gathered}
v^{\prime \prime}+A v=-w^{\prime \prime}-\frac{d}{d t} G(v+w)+f(v+w), \text { on }(0, T), \\
v(0)=u^{0}-x h(0), \quad v^{\prime}(0)=u^{1}-x h^{\prime}(0)
\end{gathered}
$$

Thus, by the variation of constants formula, we have

$$
\begin{aligned}
v(t) & =C(t)\left(u^{0}-x h(0)\right)+S(t)\left(u^{1}-x h^{\prime}(0)\right) \\
& +\int_{0}^{t} S(t-\tau)\left[f(u(\tau))-\frac{d}{d \tau} G(u(\tau))-w^{\prime \prime}(\tau)\right] d \tau .
\end{aligned}
$$

Formal integration by parts yields

$$
\begin{aligned}
u(t) & =C(t)\left(u^{0}-x h(0)\right)+S(t)\left(G\left(u^{0}\right)+u^{1}\right)+x h(t) \\
& -\int_{0}^{t} C(t-\tau) x h^{\prime}(\tau) d \tau+\int_{0}^{t} S(t-\tau) f(u(\tau)) d \tau \\
& -\int_{0}^{t} C(t-\tau) G(u(\tau)) d \tau .
\end{aligned}
$$


By differentiating (2.7), one has

$$
\begin{aligned}
u^{\prime}(t) & =C(t)\left(G\left(u^{0}\right)+u^{1}\right)-A S(t)\left(u^{0}-x h(0)\right. \\
& +\int_{0}^{t} A S(t-\tau) x h^{\prime}(\tau) d \tau+\int_{0}^{t} C(t-\tau) f(u(\tau)) d \tau \\
& +\int_{0}^{t} A S(t-\tau) G(u(\tau)) d \tau-G(u(t)) .
\end{aligned}
$$

At this end we let

$$
\begin{aligned}
U_{0}(t) & =C(t)\left(u^{0}-x h(0)\right)+S(t)\left(G\left(u^{0}\right)+u^{1}\right)+x h(t) \\
& -\int_{0}^{t} C(t-\tau) x h^{\prime}(\tau) d \tau
\end{aligned}
$$

and

$$
\begin{aligned}
V_{0}(t) & =C(t)\left(G\left(u^{0}\right)+u^{1}\right)-A S(t)\left(u^{0}-x h(0)\right) \\
& +\int_{0}^{t} A S(t-\tau) x h^{\prime}(\tau) d \tau
\end{aligned}
$$

The following regularity results are well known (for example, see [13, 14]), and thus their proofs are omitted.

Lemma 2.3. For $s \geq 0$ we have

(i) $C(\cdot) \in \mathcal{L}\left(\mathcal{D}\left(A^{s}\right), C\left([0, T], \mathcal{D}\left(A^{s}\right)\right)\right)$,

(ii) $S(\cdot) \in \mathcal{L}\left(\mathcal{D}\left(A^{s}\right), C\left([0, T], \mathcal{D}\left(A^{s+1 / 2}\right)\right)\right)$,

Remark 2.4. In view of Lemma 2.1, Lemma 2.3 the assumptions on $u^{0}, u^{1}$, and the fact that $w \in C^{1}\left([0, \infty), \mathcal{D}\left(A^{1 / 2}\right)\right)$, it is easy to verify that $U_{0} \in C\left([0, \infty), H_{0,0}^{1}\right)$ and $V_{0} \in C\left([0, \infty), L^{2}(0,1)\right)$. Also, it is not too difficult to show that if $u \in$ $L^{2}\left(0, T, H_{0,0}^{1}\right), u^{\prime} \in L^{2}\left(0, T, L^{2}(0,1)\right)$, and $u$ satisfies the integral equations

$$
\begin{aligned}
u(t)= & U_{0}(t)+\int_{0}^{t} S(t-\tau) f(u(\tau)) d \tau-\int_{0}^{t} C(t-\tau) G(u(\tau)) d \tau \\
u^{\prime}(t) & =V_{0}(t)+\int_{0}^{t} C(t-\tau) f(u(\tau)) d \tau+\int_{0}^{t} A S(t-\tau) G(u(\tau)) d \tau \\
& -G(u(t))
\end{aligned}
$$

then $u$ is a weak solution to (1.1) in the sense of Definition 2.2. Moreover, the converse is also valid. The proof of this remark is similar to that of Remark 2.1 in [2], and thus it is omitted.

\section{Local Existence}

Our first step is to establish the existence of a local weak solution to (1.1), without any restriction on the damping or source terms. We accomplish this by constructing a sequence of approximate solutions to (1.1) and obtaining the necessary estimates for the passage to the limit. Let $\left\{e_{k}\right\}_{k=1}^{\infty}$ be the orthonormal basis for $L^{2}(0,1)$, as described in Section 2, Let $\mathcal{P}_{N}$ be the orthogonal projection of $L^{2}(0,1)$ onto the linear span of $\left\{e_{1}, \ldots, e_{N}\right\}$. Let $u_{N}(t)=\sum_{k=1}^{N} u_{N, k}(t) e_{k}$ be a weak solution 
to the Galerkin system associated with the initial-boundary value problem (1.1), i.e., $u_{N}(t)$ satisfies the initial value problem

$$
\begin{gathered}
\frac{d}{d t}\left\langle u_{N}^{\prime}(t), e_{k}\right\rangle_{L^{2}(0,1)}+\left\langle A^{1 / 2} u_{N}(t), A^{1 / 2} e_{k}\right\rangle_{L^{2}(0,1)}-\sqrt{2}(-1)^{k+1} h(t) \\
-\left\langle\mathcal{P}_{N} f\left(u_{N}(t)\right), e_{k}\right\rangle_{L^{2}(0,1)}+\left\langle\mathcal{P}_{N} \frac{d}{d t} G\left(u_{N}(t)\right), e_{k}\right\rangle_{L^{2}(0,1)}=0 \\
u_{N, k}(0)=u_{k}^{0}, \quad u_{N, k}^{\prime}(0)=u_{k}^{1},
\end{gathered}
$$

for $k=1,2, \ldots, N$, where $u_{k}^{0}=\left\langle u^{0}, e_{k}\right\rangle_{L^{2}(0,1)}$ and $u_{k}^{1}=\left\langle u^{1}, e_{k}\right\rangle_{L^{2}(0,1)}$.

Now, (3.1) and (3.2) are equivalent to

$$
\begin{aligned}
u_{N, k}^{\prime \prime}(t)+\lambda_{k} u_{N, k}(t) & =\sqrt{2}(-1)^{k+1} h(t)+\left\langle\mathcal{P}_{N} f\left(u_{N}(t)\right), e_{k}\right\rangle_{L^{2}(0,1)} \\
& -\left\langle\mathcal{P}_{N} \frac{d}{d t} G\left(u_{N}(t)\right), e_{k}\right\rangle_{L^{2}(0,1)} \\
u_{N, k}(0) & =u_{k}^{0}, \quad u_{N, k}^{\prime}(0)=u_{k}^{1},
\end{aligned}
$$

for $k=1,2, \ldots, N$.

Note that (3.3)-(3.4) is an initial value problem for a second order $N \times N$ system of ordinary differential equations. Since the nonlinearities $f$ and $G$ are locally Lipschitz, it follows from the theory of ordinary differential equations that (3.3)(3.4) has a unique solution $u_{N, k}$ on the interval $\left[0, T_{N}\right]$, for some $T_{N}>0$. Moreover, $u_{N, k} \in C^{2}\left[0, T_{N}\right]$, and they satisfy the following integral equation on $\left[0, T_{N}\right]$ :

$$
\begin{aligned}
u_{N, k}(t) & =u_{k}^{0} \cos \lambda_{k}^{1 / 2} t+u_{k}^{1} \lambda_{k}^{-1 / 2} \sin \lambda_{k}^{1 / 2} t \\
& +\int_{0}^{t} \lambda_{k}^{-1 / 2} \sin \lambda_{k}^{1 / 2}(t-\tau) H_{N, k}(\tau) d \tau,
\end{aligned}
$$

for $k=1,2, \ldots, N$, where

$$
\begin{aligned}
H_{N, k}(\tau) & =\sqrt{2}(-1)^{k+1} h(\tau)+\left\langle\mathcal{P}_{N} f\left(u_{N}(\tau)\right), e_{k}\right\rangle_{L^{2}(0,1)} \\
& -\left\langle\mathcal{P}_{N} \frac{d}{d \tau} G\left(u_{N}(\tau)\right), e_{k}\right\rangle_{L^{2}(0,1)} .
\end{aligned}
$$

By integration by parts in the left-hand side of (3.5) (the integrals involving the first and third terms of $\left.H_{N, k}(\tau)\right)$, and recognizing the simple fact that $\sqrt{2}(-1)^{k+1}=$ $\lambda_{k}\left\langle x, e_{k}\right\rangle_{L^{2}(0,1)}$, it follows from the definitions of the sine and cosine operators that $u_{N}$ satisfies the following integral equations on $\left[0, T_{N}\right]$ :

$$
\begin{aligned}
u_{N}(t) & =U_{N, 0}(t)+\int_{0}^{t} S(t-\tau) \mathcal{P}_{N} f\left(u_{N}(\tau)\right) d \tau \\
& -\int_{0}^{t} C(t-\tau) \mathcal{P}_{N} G\left(u_{N}(\tau)\right) d \tau \\
u_{N}^{\prime}(t)= & V_{N, 0}(t)+\int_{0}^{t} C(t-\tau) \mathcal{P}_{N} f\left(u_{N}(\tau)\right) d \tau \\
+ & \int_{0}^{t} A S(t-\tau) \mathcal{P}_{N} G\left(u_{N}(\tau)\right) d \tau-\mathcal{P}_{N} G\left(u_{N}(t)\right),
\end{aligned}
$$


where

$$
\begin{aligned}
U_{N, 0}(t) & =C(t) \mathcal{P}_{N}\left(u^{0}-x h(0)\right)+S(t)\left(\mathcal{P}_{N} G\left(\mathcal{P}_{N} u^{0}\right)+\mathcal{P}_{N} u^{1}\right) \\
& +\mathcal{P}_{N} x h(t)-\int_{0}^{t} \mathcal{P}_{N} C(t-\tau) x h^{\prime}(\tau) d \tau
\end{aligned}
$$

and

$$
\begin{aligned}
V_{N, 0}(t) & =C(t)\left(\mathcal{P}_{N} G\left(\mathcal{P}_{N} u^{0}\right)+\mathcal{P}_{N} u^{1}\right)-A S(t) \mathcal{P}_{N}\left(u^{0}-x h(0)\right) \\
& +\int_{0}^{t} \mathcal{P}_{N} A S(t-\tau) x h^{\prime}(\tau) d \tau .
\end{aligned}
$$

A priori estimates: Here, we shall show that, for all $N, T_{N}$ can be replaced by some $T>0$. Also, in the rest of the paper, we shall refer to the following Hilbert spaces repeatedly:

$$
X=L^{2}\left(0, T, L^{2}(0,1)\right) \text { and } Y=L^{2}\left(0, T, H_{0,0}^{1}\right)
$$

Lemma 3.1. There exists a constant $T>0$ such that the sequence of approximate solutions $u_{N}$ satisfies the following:

(i) $\left\{u_{N}\right\}$ is bounded in $Y$;

(ii) $\left\{u_{N}^{\prime}\right\}$ is bounded in $X$.

Proof. Fix $T_{0}>0$. First note that by using Lemma 2.2, it is easy to check that $U_{N, 0} \in C\left(\left[0, T_{0}\right], H_{0,0}^{1}\right)$ and $V_{N, 0} \in C\left(\left[0, T_{0}\right], L^{2}(0,1)\right)$. Also, we note that $\mathcal{P}_{N} u^{0} \rightarrow$ $u^{0}$ in $H_{0,0}^{1}$, and $\mathcal{P}_{N} u^{1} \rightarrow u^{1}$ in $L^{2}(0,1)$. Therefore, it follows from Lemmas 2.1 and 2.2 that $U_{N, 0} \rightarrow U_{0}$ in $C\left(\left[0, T_{0}\right], H_{0,0}^{1}\right)$ and $V_{N, 0} \rightarrow V_{0}$ in $C\left(\left[0, T_{0}\right], L^{2}(0,1)\right)$ as $N \rightarrow \infty$. So, there exists a constant $M>0$ such that, for all $N$,

$$
\sup _{t \in\left[0, T_{0}\right]}\left\|U_{N, 0}(t)\right\|_{H_{0,0}^{1}} \leq \sup _{t \in\left[0, T_{0}\right]}\left\|U_{0}(t)\right\|_{H_{0,0}^{1}}+M \equiv \alpha
$$

and

$$
\sup _{t \in\left[0, T_{0}\right]}\left\|V_{N, 0}(t)\right\|_{L^{2}(0,1)} \leq \sup _{t \in\left[0, T_{0}\right]}\left\|V_{0}(t)\right\|_{L^{2}(0,1)}+M \equiv \beta
$$

We remark here that in (3.11) and (3.12), the constants $\alpha$ and $\beta$ depend on $T_{0}$. It follows from (3.7) and Lemma 2.3 that, for all $t \in\left[0, T_{0}\right]$ and all $N \geq 1$,

$$
\begin{aligned}
\left\|u_{N}(t)\right\|_{H_{0,0}^{1}} \leq & \left\|U_{N, 0}(t)\right\|_{H_{0,0}^{1}}+\int_{0}^{t}\left\|S(t-\tau) \mathcal{P}_{N} f\left(u_{N}(\tau)\right)\right\|_{H_{0,0}^{1}} d \tau \\
& +\int_{0}^{t}\left\|C(t-\tau) \mathcal{P}_{N} G\left(u_{N}(\tau)\right)\right\|_{H_{0,0}^{1}} d \tau \\
\leq \quad & \alpha+\int_{0}^{t}\left\|\left|u_{N}(\tau)\right|^{p-1} u_{N}(\tau)\right\|_{L^{2}(0,1)} d \tau \\
& +\int_{0}^{t}\left\|G\left(u_{N}(\tau)\right)\right\|_{H_{0,0}^{1}} d \tau
\end{aligned}
$$

Since $H_{0,0}^{1} \hookrightarrow C^{0}[0,1]$, with $\|u(\tau)\|_{\infty} \leq\|u(\tau)\|_{H_{0,0}^{1}}$, one has

$$
\left\|\left|u_{N}(\tau)\right|^{p-1} u_{N}(\tau)\right\|_{L^{2}(0,1)} \leq\left\|u_{N}(\tau)\right\|_{\infty}^{p} \leq\left\|u_{N}(\tau)\right\|_{H_{0,0}^{1}}^{p} .
$$


Moreover,

$$
\begin{aligned}
\left\|G\left(u_{N}(\tau)\right)\right\|_{H_{0,0}^{1}}^{2} & \leq \int_{0}^{1}\left(\left|u_{N}(\tau)\right|^{2 m}+\left.\left.|| u_{N}(\tau)\right|^{m-1}\left(u_{N}\right)_{x}(\tau)\right|^{2}\right) d x \\
& \leq\left\|u_{N}(\tau)\right\|_{H_{0,0}^{1}}^{2 m}+\left\|u_{N}(\tau)\right\|_{\infty}^{2(m-1)}\left\|u_{N}(\tau)\right\|_{H_{0,0}^{1}}^{2} \\
& \leq 2\left\|u_{N}(\tau)\right\|_{H_{0,0}^{1}}^{2 m} .
\end{aligned}
$$

Therefore, for $t \in\left[0, T_{0}\right]$ we have

$$
\left\|u_{N}(t)\right\|_{H_{0,0}^{1}} \leq \alpha+\int_{0}^{t}\left(\left\|u_{N}(\tau)\right\|_{H_{0,0}^{1}}^{p}+2\left\|u_{N}(\tau)\right\|_{H_{0,0}^{1}}^{m}\right) d \tau .
$$

Let $1+\left\|u_{N}(t)\right\|_{H_{0,0}^{1}}:=y_{N}(t)$ and $\sigma=\max (p, m)$. Then, for all $t \in\left[0, T_{0}\right]$ we have

$$
y_{N}(t) \leq 1+\alpha+3 \int_{0}^{t} y_{N}(\tau)^{\sigma} d \tau
$$

It follows from $(3.17)$ that $y_{N}(t) \leq Y(t)$, where $Y(t)=\left[(1+\alpha)^{1-\sigma}-3(\sigma-1) t\right]^{\frac{-1}{\sigma-1}}$ is the solution to the Volterra integral equation

$$
Y(t)=1+\alpha+3 \int_{0}^{t} Y(\tau)^{\sigma} d \tau
$$

Although $Y(t)$ blows up in finite time (since $\sigma>1$ and $\alpha>0$ ), there exists a time $T>0, T<T_{0}$ such that $y_{N}(t) \leq Y(t) \leq C$ for all $t \in[0, T]$ and all $N \geq 1$. This shows that $\left\{u_{N}\right\}$ is bounded in $Y$.

Now, it follows from (3.8), Lemma 2.3 and (3.15) that, for all $t \in[0, T]$ and all $N \geq 1$,

$$
\begin{aligned}
\left\|u_{N}^{\prime}(t)\right\|_{L^{2}(0,1)} \leq & \left\|V_{0, N}(t)\right\|_{L^{2}(0,1)}+\left\|\left|u_{N}(t)\right|^{m-1} u_{N}(t)\right\|_{L^{2}(0,1)} \\
& +\int_{0}^{t}\left\|\left|u_{N}(\tau)\right|^{p-1} u_{N}(\tau)\right\|_{L^{2}(0,1)} d \tau \\
& +\int_{0}^{t}\left\|A S(t-\tau) \mathcal{P}_{N} G\left(u_{N}(\tau)\right)\right\|_{L^{2}(0,1)} d \tau \\
\leq & \beta+\left\|u_{N}(t)\right\|_{H_{0,0}^{1}}^{m}+\int_{0}^{t}\left\|u_{N}(\tau)\right\|_{H_{0,0}^{1}}^{p} d \tau \\
& +\int_{0}^{t}\left\|G\left(u_{N}(\tau)\right)\right\|_{H_{0,0}^{1}} d \tau \\
\leq & \beta+\left\|u_{N}(t)\right\|_{H_{0,0}^{1}}^{m} \\
& +\int_{0}^{t}\left(\left\|u_{N}(\tau)\right\|_{H_{0,0}^{1}}^{p}+2\left\|u_{N}(\tau)\right\|_{H_{0,0}^{1}}^{m}\right) d \tau .
\end{aligned}
$$

Since $\left\{u_{N}(t)\right\}$ is a bounded sequence in $H_{0,0}^{1}$ for every $t \in[0, T]$, then $\left\{u_{N}^{\prime}\right\}$ is bounded in $X$.

The following compactness theorem is a special case of a more general theorem, which can be found in [24], for example. 
Compactness Theorem. Let $X$ and $Y$ be the Hilbert spaces described above. Let $\mathcal{Y}$ be the space of functions $\mathcal{Y}=\left\{u \in Y, u^{\prime} \in X\right\}$ endowed with the natural norm $\|u\|_{\mathcal{Y}}^{2}=\|u\|_{Y}^{2}+\|u\|_{X}^{2}$. Then the imbedding $\mathcal{Y} \stackrel{i}{\hookrightarrow} X$ is compact.

Now, by using Lemma 3.1 and the above compactness theorem, we can extract a subsequence of $\left\{u_{N}\right\}$ (still denoted by $\left\{u_{N}\right\}$ ) and find a function $u \in Y$ with $u^{\prime} \in X$ such that

$$
\left\{\begin{array}{l}
u_{N} \rightarrow u \text { strongly in } X \\
u_{N} \rightarrow u \text { weakly in } Y, \\
u_{N}(t) \rightarrow u(t) \text { strongly in } L^{2}(0,1) \text { for almost all } t \in[0, T], \\
u_{N}^{\prime} \rightarrow u^{\prime} \text { weakly in } X, \\
u_{N}^{\prime}(t) \rightarrow u^{\prime}(t) \text { weakly in } L^{2}(0,1) \text { for almost all } t \in[0, T] .
\end{array}\right.
$$

Before passing to the limit we prove the following lemma.

Lemma 3.2. There exists a subsequence of the approximate solutions in (3.20), still denoted by $u_{N}$, that satisfies

$$
f\left(u_{N}(t)\right) \rightarrow f(u(t)) \text { and } G\left(u_{N}(t)\right) \rightarrow G(u(t)) \text { in } L^{2}(0,1),
$$

for almost all $t \in[0, T]$.

Proof. First we remark that $\left\|u_{N}(t)\right\|_{\infty} \leq\left\|u_{N}(t)\right\|_{H_{0,0}^{1}} \leq C$, for all $t \in[0, T]$. Therefore, for almost all $t \in[0, T]$, we have

$$
\begin{aligned}
& \left\|f\left(u_{N}(t)\right)-f(u(t))\right\|_{L^{2}(0,1)}=\left\|\left|u_{N}(t)\right|^{p-1} u_{N}(t)-|u(t)|^{p-1} u(t)\right\|_{L^{2}(0,1)} \\
& \leq\left\|\left|u_{N}(t)\right|^{p-1} u_{N}(t)-\left|u_{N}(t)\right|^{p-1} u(t)\right\|_{L^{2}(0,1)} \\
& \quad+\left\|\left|u_{N}(t)\right|^{p-1} u_{N}(t)-|u(t)|^{p-1} u(t)\right\|_{L^{2}(0,1)} \\
& \leq C\left(\left\|u_{N}(t)-u(t)\right\|_{L^{2}(0,1)}+\left\|\left|u_{N}(t)\right|^{p-1}-|u(t)|^{p-1}\right\|_{L^{2}(0,1)}\right) .
\end{aligned}
$$

Since $u_{N} \rightarrow u$ strongly in $X=L^{2}\left(0, T, L^{2}(0,1)\right)$, then there exists a subsequence of $\left\{u_{N}\right\}$ (still denoted $\left\{u_{N}\right\}$ ) such that $u_{N}(x, t) \rightarrow u(x, t)$ for almost every $(x, t) \in(0,1) \times(0, T)$. It follows from the dominated convergence theorem that $\left\|\left|u_{N}(t)\right|^{p-1}-|u(t)|^{p-1}\right\|_{L^{2}(0,1)} \rightarrow 0$, for almost all $t \in[0, T]$. Therefore, (3.21) yields that $\left\|f\left(u_{N}(t)\right)-f(u(t))\right\|_{L^{2}(0,1)} \rightarrow 0$.

Now, the sequence of approximate solutions in Lemma 3.2 satisfies

$$
\begin{aligned}
\left\langle u_{N}^{\prime}(t), e_{k}\right\rangle_{L^{2}(0,1)}-\left\langle\mathcal{P}_{N} u^{1}, e_{k}\right\rangle_{L^{2}(0,1)}+\int_{0}^{t}\left\langle A^{1 / 2} u_{N}(s), A^{1 / 2} e_{k}\right\rangle_{L^{2}(0,1)} d s \\
\quad-\int_{0}^{t}\left[h(s) e_{k}(1)+\left\langle\mathcal{P}_{N} f\left(u_{N}(s), e_{k}\right\rangle_{L^{2}(0,1)}\right] d s\right. \\
+\left\langle\mathcal{P}_{N} G\left(u_{N}(t), e_{k}\right\rangle_{L^{2}(0,1)}-\left\langle\mathcal{P}_{N} G\left(\mathcal{P}_{N} u^{0}\right), e_{k}\right\rangle_{L^{2}(0,1)}=0,\right.
\end{aligned}
$$

for $k=1,2, \ldots, N$ and almost every $t \in[0, T]$. 
By letting $N \rightarrow \infty$, using (3.20), Lemma 3.2 , and the fact that the trace operator $\phi \rightarrow \phi(1)$ is continuous on $H_{0,0}^{1}$, one finds that the limit function $u$ satisfies $(2.3)$ on the interval $[0, T]$, i.e., $u$ is a weak solution to $(1.1)$ on $[0, T]$. In view of Remark 2.4, then $u$ satisfies the integral equations (2.11)-(2.12). Therefore, we deduce that $u \in C\left([0, T], H_{0,0}^{1}\right)$ and $u^{\prime} \in C\left([0, T], L^{2}(0,1)\right)$. So we have proven the following theorem.

Theorem 3.3. Let $u^{0}, u^{1}$, and $h$ satisfy the assumptions (H1) and (H2). Then, there exists a constant $T>0$ such that the initial-boundary value problem (1.1) has a unique weak solution $u$ on $[0, T]$, where

$$
u \in C\left([0, T], H_{0,0}^{1}\right) \text { and } u^{\prime} \in C\left([0, T], L^{2}(0,1)\right) .
$$

Lemma 3.4. Let $u$ be a weak solution to the initial-boundary value problem (1.1) as given in Theorem 3.3. Then $u^{\prime \prime} \in L^{2}\left(0, T,\left(H_{0,0}^{1}\right)^{\prime}\right)$.

Proof. Let $\langle\cdot, \cdot\rangle$ denote the standard pairing of $\left(H_{0,0}^{1}\right)^{\prime}$ and $H_{0,0}^{1}$. Then for all $\phi \in H_{0,0}^{1}$ and almost all $t \in[0, T]$,

$$
\begin{gathered}
\left|<u^{\prime \prime}(t), \phi\right\rangle|=| \frac{d}{d t}\left\langle u^{\prime}(t), \phi\right\rangle_{L^{2}}|\leq|\left\langle A^{1 / 2} u(t), A^{1 / 2} \phi\right\rangle_{L^{2}} \mid \\
+\quad|h(t)||\phi(1)|+\left|\langle f(u(t)), \phi\rangle_{L^{2}}\right|+\left|\left\langle\frac{d}{d t} G(u(t)), \phi\right\rangle_{L^{2}}\right| \\
\leq \quad\left(\|u(t)\|_{H_{0,0}^{1}}+C(h, T)+\|f(u(t))\|_{L^{2}}+\|\left.\frac{d}{d t} G(u(t))\right|_{L^{2}}\right)\|\phi\|_{H_{0,0}^{1}} \\
\leq \quad\left(\|u\|_{C\left([0, T], H_{0,0}^{1}\right)}+C(h, T)+\|u\|_{C\left([0, T], H_{0,0}^{1}\right)}^{p}\right. \\
\left.+\|u\|_{C\left([0, T], H_{0,0}^{1}\right)}^{m-1}\left\|u^{\prime}\right\|_{C\left([0, T], L^{2}\right)}\right)\|\phi\|_{H_{0,0}^{1}} \leq C(h, T)\|\phi\|_{H_{0,0}^{1}} .
\end{gathered}
$$

Hence, $u^{\prime \prime} \in L^{2}\left(0, T,\left(H_{0,0}^{1}\right)^{\prime}\right)$.

\section{Global Solutions}

We begin by deriving an energy identity for the approximate solutions $\left\{u_{N}\right\}$. By multiplying equation (3.3) by $u_{N, k}^{\prime}(t)$, summing from 1 to $N$, and integrating from 0 to $t$, one has

$$
E_{N}(t)=E_{N}(0) \text { for } t \in[0, T]
$$

where

$$
\begin{aligned}
E_{N}(t)= & \frac{1}{2}\left(\left\|u_{N}^{\prime}(t)\right\|_{L^{2}(0,1)}^{2}+\left\|A^{1 / 2} u_{N}(t)\right\|_{L^{2}(0,1)}^{2}\right) \\
& -\frac{1}{p+1}\left\|u_{N}(x, t)\right\|_{L^{p+1}(0,1)}^{p+1}+\int_{0}^{t} \int_{0}^{1}\left|u_{N}(\tau)\right|^{m-1} u_{N}^{\prime}(\tau)^{2} d x d \tau \\
& -h(t) u_{N}(1, t)+h(0) u_{N}^{0}(1)+\int_{0}^{t} h^{\prime}(\tau) u_{N}(1, \tau) d \tau
\end{aligned}
$$

Due to the fact that the solution of the initial-boundary value problem (1.1) is not sufficiently regular, obtaining the energy identity in Lemma 4.1 is not straightforward. However, by modifying the proof of Lemma 8.3 of Lions and Magenes [19], Lemma 4.1 follows. Thus, its proof is omitted. 
Lemma 4.1. Let $u \in C\left([0, T], H_{0,0}^{1}\right)$ and $u^{\prime} \in C\left([0, T], L^{2}(0,1)\right)$ be such that $u$ is a weak solution to the initial-boundary value problem (1.1). Then, u satisfies

$$
E(t)=E(0)
$$

where

$$
\begin{aligned}
E(t)= & \frac{1}{2}\left(\left\|u^{\prime}(t)\right\|_{L^{2}(0,1)}^{2}+\left\|A^{1 / 2} u(t)\right\|_{L^{2}(0,1)}^{2}\right) \\
& -\frac{1}{p+1}\|u(x, t)\|_{L^{p+1}(0,1)}^{p+1}+\int_{0}^{t} \int_{0}^{1}|u(\tau)|^{m-1} u^{\prime}(\tau)^{2} d x d \tau \\
& -h(t) u(1, t)+h(0) u^{0}(1)+\int_{0}^{t} h^{\prime}(\tau) u(1, \tau) d \tau .
\end{aligned}
$$

Theorem 4.2. Let $m \geq p$. Then for any $T>0$, the initial-boundary value problem (1.1) has a unique weak solution $u$ on $[0, T]$, where

$$
u \in C\left([0, T], H_{0,0}^{1}\right) \text { and } u^{\prime} \in C\left([0, T], L^{2}(0,1)\right) .
$$

Proof. The theorem will follow immediately from Lemma 4.3 below. The a priori bounds for the approximate solutions in Lemma 4.3. followed by application of (3.20) and Lemma 3.2. allow us to pass to the limit and obtain a weak solution to (1.1) on any time interval $[0, T]$.

Lemma 4.3. If $m \geq p$, then, on any bounded time interval $[0, T],\left\{u_{N}\right\}$ is bounded in $Y$ and $\left\{u_{N}^{\prime}\right\}$ is bounded in $X$.

Proof. Let

$$
\begin{gathered}
e_{N}(t)=\frac{1}{2}\left(\left\|u_{N}^{\prime}(t)\right\|_{L^{2}(0,1)}^{2}+\left\|A^{1 / 2} u_{N}(t)\right\|_{L^{2}(0,1)}^{2}\right), \\
F_{N}(t)=e_{N}(t)+\frac{1}{p+1}\left\|u_{N}(t)\right\|_{p+1}^{p+1}
\end{gathered}
$$

and

$$
\sigma_{N}(t)=h(t) u_{N}(1, t)-h(0) u_{N}^{0}(1)-\int_{0}^{t} h^{\prime}(\tau) u_{N}(1, \tau) d \tau .
$$

We shall show that $F_{N}(t)$ remains bounded on bounded time intervals. It follows from (4.2) that

$$
\begin{aligned}
e_{N}^{\prime}(t)=\int_{0}^{1}\left|u_{N}(t)\right|^{p-1} & u_{N}(t) u_{N}^{\prime}(t) d x \\
& -\int_{0}^{1}\left|u_{N}(t)\right|^{m-1} u_{N}^{\prime}(t)^{2} d x+\sigma_{N}^{\prime}(t) .
\end{aligned}
$$

Therefore,

$$
\begin{aligned}
F_{N}^{\prime}(t)=2 \int_{0}^{1}\left|u_{N}(t)\right|^{p-1} u_{N}(t) u_{N}^{\prime}(t) d x \\
\\
-\int_{0}^{1}\left|u_{N}(t)\right|^{m-1} u_{N}^{\prime}(t)^{2} d x+\sigma_{N}^{\prime}(t) .
\end{aligned}
$$


However, the Cauchy-Schwarz inequality yields

$$
\begin{aligned}
& \left.\left|\int_{0}^{1}\right| u_{N}(t)\right|^{p-1} u_{N}(t) u_{N}^{\prime}(t) d x \mid \\
& \quad \leq\left(\int_{0}^{1}\left|u_{N}(t)\right|^{p+1} d x\right)^{1 / 2}\left(\int_{0}^{1}\left|u_{N}(t)\right|^{p-1}\left|u_{N}^{\prime}(t)\right|^{2} d x\right)^{1 / 2} \\
& \quad \leq \frac{1}{2}\left(\int_{0}^{1}\left|u_{N}(t)\right|^{p+1} d x+\int_{0}^{1}\left|u_{N}(t)\right|^{p-1}\left|u_{N}^{\prime}(t)\right|^{2} d x\right) .
\end{aligned}
$$

Therefore, one has

$$
\begin{aligned}
F_{N}^{\prime}(t) \leq & \int_{0}^{1}\left|u_{N}(t)\right|^{p+1} d x+\int_{0}^{1}\left|u_{N}(t)\right|^{p-1}\left|u_{N}^{\prime}(t)\right|^{2} d x \\
& -\int_{0}^{1}\left|u_{N}(t)\right|^{m-1} u_{N}^{\prime}(t)^{2} d x+\sigma_{N}^{\prime}(t) \\
= & \int_{0}^{1} u_{N}^{\prime}(t)^{2}\left(\left|u_{N}(t)\right|^{p-1}-\left|u_{N}(t)\right|^{m-1}\right) d x \\
& +\int_{0}^{1}\left|u_{N}(t)\right|^{p+1} d x+\sigma_{N}^{\prime}(t) \\
= & I_{N}^{1}(t)+I_{N}^{2}(t)+\int_{0}^{1}\left|u_{N}(t)\right|^{p+1} d x+\sigma_{N}^{\prime}(t),
\end{aligned}
$$

where

$$
I_{N}^{1}(t)=\int_{\left\{x \in[0,1]:\left|u_{N}\right|>1\right\}} u_{N}^{\prime}(t)^{2}\left(\left|u_{N}(t)\right|^{p-1}-\left|u_{N}(t)\right|^{m-1}\right) d x
$$

and

$$
I_{N}^{2}(t)=\int_{\left\{x \in[0,1]: 0 \leq\left|u_{N}\right| \leq 1\right\}} u_{N}^{\prime}(t)^{2}\left(\left|u_{N}(t)\right|^{p-1}-\left|u_{N}(t)\right|^{m-1}\right) d x .
$$

Since $m \geq p$, then $I_{N}^{1}(t) \leq 0$ and $I_{N}^{2}(t) \leq 2 \int_{0}^{1} u_{N}^{\prime}(t)^{2} d x$. It follows from (4.11) that

$$
\begin{aligned}
F_{N}^{\prime}(t) & \leq 2 \int_{0}^{1} u_{N}^{\prime}(t)^{2} d x+\int_{0}^{1}\left|u_{N}(t)\right|^{p+1} d x+\sigma_{N}^{\prime}(t) \\
& \leq 4(p+1) F_{N}(t)+\sigma_{N}^{\prime}(t) .
\end{aligned}
$$

By multiplying (4.12) by $e^{-4(p+1) t}$ and integrating from 0 to $t$, we obtain

$$
\begin{aligned}
F_{N}(t) \leq & F_{N}(0) e^{4(p+1) t}+\sigma_{N}(t) \\
& +4(p+1) e^{4(p+1) t} \int_{0}^{t} e^{-4(p+1) \tau} \sigma_{N}(\tau) d \tau .
\end{aligned}
$$

However,

$$
\begin{aligned}
F_{N}(0) & =\frac{1}{2}\left(\left\|u_{N}^{1}\right\|_{L^{2}}^{2}+\left\|A^{1 / 2} u_{N}^{0}\right\|_{L^{2}}^{2}\right)+\frac{1}{p+1}\left\|u_{N}^{0}\right\|_{L^{p+1}}^{p+1} \\
& \leq\left\|u^{1}\right\|_{L^{2}}^{2}+\left\|u^{0}\right\|_{H_{0,0}^{1}}^{2}+\left\|u_{N}^{0}\right\|_{\infty}^{p+1} \\
& \leq\left\|u^{1}\right\|_{L^{2}}^{2}+\left\|u^{0}\right\|_{H_{0,0}^{1}}^{2}+\left\|u^{0}\right\|_{H_{0,0}^{1}}^{p+1}:=C_{0},
\end{aligned}
$$

where $C_{0}$ depends only on the initial data $u^{0}$ and $u^{1}$. 
Now, on any bounded time interval $[0, T]$, we estimate $\sigma_{N}(t)$ as follows:

$$
\begin{aligned}
\left|\sigma_{N}(t)\right| \leq & |h(t)|\left|u_{N}(1, t)\right|+|h(0)|\left|u_{N}^{0}(1)\right|+\int_{0}^{t}\left|h^{\prime}(\tau)\right|\left|u_{N}(1, \tau)\right| d \tau \\
\leq & |h(t)|^{2}+\frac{1}{4}\left|u_{N}(1, t)\right|^{2}+|h(0)|\left\|u_{N}^{0}\right\|_{H_{0,0}^{1}} \\
& +\frac{1}{2} \int_{0}^{t}\left|h^{\prime}(\tau)\right|^{2} d \tau+\frac{1}{2} \int_{0}^{t}\left\|u_{N}(\tau)\right\|_{H_{0,0}^{1}}^{2} d \tau \\
\leq & C\left(h, T, u^{0}\right)+\frac{1}{4}\left\|u_{N}(t)\right\|_{H_{0,0}^{1}}^{2}+\frac{1}{2} \int_{0}^{t}\left\|u_{N}(\tau)\right\|_{H_{0,0}^{1}}^{2} d \tau \\
\leq & C+\frac{1}{2} F_{N}(t)+\int_{0}^{t} F_{N}(\tau) d \tau,
\end{aligned}
$$

where $C$ is a positive constant that depends on $h, T$ and $u^{0}$. Therefore, it follows from (4.13)-(4.15) that

$$
\begin{gathered}
F_{N}(t) \leq C_{0} e^{4(p+1) t}+C+\frac{1}{2} F_{N}(t)+\int_{0}^{t} F_{N}(\tau) d \tau \\
+4(p+1) e^{4(p+1) t} \int_{0}^{t} e^{-4(p+1) \tau}\left(C+\frac{1}{2} F_{N}(\tau)+\int_{0}^{\tau} F_{N}(s) d s\right) d \tau \\
\leq C_{0} e^{4(p+1) T}+C^{\prime}+\frac{1}{2} F_{N}(t)+2(p+2) e^{4(p+1) T} \int_{0}^{t} F_{N}(\tau) d \tau,
\end{gathered}
$$

where $C_{0}$ and $C^{\prime}$ are positive constants depending on $h, T, u^{0}$ and $u^{1}$.

Thus, it follows from (4.16) that there exist positive constants $C_{1}$ and $C_{2}$, depending on $h, T, u^{0}$ and $u^{1}$, such that

$$
F_{N}(t) \leq C_{1}+C_{2} \int_{0}^{t} F_{N}(\tau) d \tau
$$

By Gronwall's inequality,

$$
F_{N}(t) \leq C_{1}\left(1+C_{2} t e^{C_{2} t}\right)
$$

which completes the proof.

\section{Blow-up of Solutions}

In this section, we assume that $p>m$ and that $h(t) \equiv 0$. So the energy identity (4.3) becomes

$$
\begin{aligned}
E(t)= & \frac{1}{2}\left(\left\|u^{\prime}(t)\right\|_{L^{2}(0,1)}^{2}+\left\|A^{1 / 2} u(t)\right\|_{L^{2}(0,1)}^{2}\right) \\
& \quad-\frac{1}{p+1}\|u(x, t)\|_{L^{p+1}(0,1)}^{p+1}+\int_{0}^{t} \int_{0}^{1}|u(\tau)|^{m-1} u^{\prime}(\tau)^{2} d x d \tau \\
= & E(0) .
\end{aligned}
$$

As in [5], we let

$$
F(t)=\|u(t)\|_{L^{2}(0,1)}^{2}
$$

and

$$
H(t)=-\frac{1}{2}\left(\left\|u^{\prime}(t)\right\|_{L^{2}(0,1)}^{2}+\left\|A^{1 / 2} u(t)\right\|_{L^{2}(0,1)}^{2}\right)+\frac{1}{p+1}\|u(t)\|_{L^{p+1}(0,1)}^{p+1} .
$$


Throughout this section we assume $H(0)>0$.

Theorem 5.1. Let $u$ be a weak solution to (1.1) on the interval $[0, T]$ in the sense of Definition 2.2. If $p>m$ and $H(0)>0$, then $T$ is necessarily finite, i.e., u cannot be continued for all $t>0$.

Proof. First, it follows from (5.1) that

$$
H^{\prime}(t)=\int_{0}^{1}|u(t)|^{m-1} u_{t}^{2}(t) d x>0 .
$$

Therefore,

$$
0<H(0) \leq H(t) \leq \frac{1}{p+1}\|u(t)\|_{L^{p+1}(0,1)}^{p+1},
$$

for $0 \leq t<T$.

Now choose $0<\alpha=\min \left\{\frac{p-m}{p+1}, \frac{p-1}{2(p+1)}\right\}$. So $\alpha<\frac{1}{2}$. Then choose $\epsilon>0$ small enough so that $1-\alpha-\frac{\epsilon}{(p+1)^{\alpha}} \geq 0$. Later, we may need to adjust $\epsilon$ again. As in 5], we let

$$
y(t)=H(t)^{1-\alpha}+\epsilon F^{\prime}(t)
$$

Then, we have

$$
\begin{aligned}
\frac{d}{d t} y(t) & =(1-\alpha) H(t)^{-\alpha} H^{\prime}(t)+4 \epsilon\left\|u^{\prime}(t)\right\|_{L^{2}(0,1)}^{2}+4 \epsilon H(t) \\
& +2 \epsilon \frac{p-1}{p+1}\|u(t)\|_{L^{p+1}(0,1)}^{p+1}-2 \epsilon \int_{0}^{1}|u(t)|^{m-1} u(t) u_{t}(t) d x
\end{aligned}
$$

By Hölder's and Young's inequalities, one has

$$
\begin{aligned}
& \left.\left|\int_{0}^{1}\right| u(t)\right|^{m-1} u(t) u_{t}(t) d x \mid \\
& \quad \leq\left(\int_{0}^{1}|u(t)|^{m-1} u_{t}^{2}(t) d x\right)^{1 / 2}\left(\int_{0}^{1}|u(t)|^{m+1} d x\right)^{1 / 2} \\
& \quad \leq\left(\int_{0}^{1}|u(t)|^{m-1} u_{t}^{2}(t) d x\right)^{1 / 2}\left(\int_{0}^{1}|u(t)|^{p+1} d x\right)^{\frac{m+1}{2(p+1)}} \\
& \quad=H^{\prime}(t)^{1 / 2}\|u\|_{L^{p+1}(0,1)}^{\frac{m+1}{2}} \leq \frac{1}{2}\left(\frac{1}{\delta} H^{\prime}(t)+\delta\|u(t)\|_{L^{p+1}(0,1)}^{m+1}\right)
\end{aligned}
$$

where $\delta>0$ is to be chosen later.

It follows from (5.3) and (5.4) that

$$
\begin{aligned}
& \frac{d}{d t} y(t) \geq(1-\alpha) H(t)^{-\alpha} H^{\prime}(t)+4 \epsilon\left\|u^{\prime}(t)\right\|_{L^{2}(0,1)}^{2} \\
& +4 \epsilon H(t)+2 \epsilon \frac{p-1}{p+1}\|u(t)\|_{L^{p+1}(0,1)}^{p+1}-\frac{\epsilon}{\delta} H^{\prime}(t)-\epsilon \delta\|u(t)\|_{L^{p+1}(0,1)}^{m+1} .
\end{aligned}
$$

By choosing $\delta=\frac{p-1}{p+1}\|u(t)\|_{L^{p+1}(0,1)}^{p-m}$, we get

$$
\epsilon \frac{p-1}{p+1}\|u(t)\|_{L^{p+1}(0,1)}^{p+1}-\epsilon \delta\|u(t)\|_{L^{p+1}(0,1)}^{m+1} \geq 0 .
$$


Therefore, we have

$$
\begin{aligned}
\frac{d}{d t} y(t) & \geq\left[(1-\alpha) H(t)^{-\alpha}-\frac{\epsilon}{\delta}\right] H^{\prime}(t)+4 \epsilon\left\|u^{\prime}(t)\right\|_{L^{2}(0,1)}^{2} \\
& +4 \epsilon H(t)+\epsilon \frac{p-1}{p+1}\|u(t)\|_{L^{p+1}(0,1)}^{p+1} .
\end{aligned}
$$

Now, since $H(t) \leq \frac{1}{p+1}\|u(t)\|_{L^{p+1}(0,1)}^{p+1}$ and $\delta=\frac{p-1}{p+1}\|u(t)\|_{L^{p+1}(0,1)}^{p-m}$, we have

$$
\begin{aligned}
& (1-\alpha) H(t)^{-\alpha}-\frac{\epsilon}{\delta}=H^{-\alpha}(t)\left[1-\alpha-\frac{\epsilon}{\delta} H(t)^{\alpha}\right] \\
& \quad \geq H(t)^{-\alpha}\left[1-\alpha-\frac{\epsilon}{(p+1)^{\alpha-1}(p-1)}\|u(t)\|_{L^{p+1}(0,1)}^{m-p+\alpha(p+1)}\right] .
\end{aligned}
$$

Furthermore, since $\|u(t)\|_{L^{p+1}(0,1)} \geq[(p+1) H(0)]^{\frac{1}{p+1}}>0$ and $\alpha$ was chosen so that $m-p+\alpha(p+1) \leq 0$, it follows that

$$
(1-\alpha) H(t)^{-\alpha}-\frac{\epsilon}{\delta} \geq H(t)^{-\alpha}\left[1-\alpha-\frac{\epsilon[(p+1) H(0)]^{\frac{m-p}{p+1}+\alpha}}{(p+1)^{\alpha-1}(p-1)}\right] .
$$

Now, if necessary, choose $\epsilon>0$ small enough so that

$$
1-\alpha-\frac{\epsilon[(p+1) H(0)]^{\frac{m-p}{p+1}+\alpha}}{(p+1)^{\alpha-1}(p-1)} \geq 0 .
$$

Therefore, it follows from (5.6), (5.8) and (5.9) that

$$
\frac{d}{d t} y(t) \geq \epsilon C\left[H(t)+\left\|u^{\prime}(t)\right\|_{L^{2}(0,1)}^{2}+\|u(t)\|_{L^{p+1}(0,1)}^{p+1}\right],
$$

for $t \in[0, T)$.

This shows that $y(t)$ is increasing on $[0, T)$, with

$$
y(t)=H(t)^{1-\alpha}+\epsilon F^{\prime}(t) \geq H(0)^{1-\alpha}+\epsilon F^{\prime}(0) .
$$

If $F^{\prime}(0) \geq 0$, then no further condition on $\epsilon$ is needed. However, if $F^{\prime}(0)<0$, then we further adjust $\epsilon$ so that $0<\epsilon<-\frac{H(0)^{1-\alpha}}{F^{\prime}(0)}$. In any case, one has $y(t)>0$, for $t \in[0, T)$.

Finally, we show that $y(t)$ satisfies the differential inequality

$$
\frac{d}{d t} y(t) \geq C_{0} y(t)^{\frac{1}{1-\alpha}} .
$$

for $t \in[0, T)$ and $C_{0}$ a positive constant.

If $F^{\prime}(t) \leq 0$ on a subset of $[0, T)$, then on this subset we have

$$
\left[H(t)^{1-\alpha}+\epsilon F^{\prime}(t)\right]^{\frac{1}{1-\alpha}} \leq H(t) .
$$

Thus, (5.10) and (5.13) show that (5.12) is valid on the subset on which $F^{\prime}(t) \leq 0$. If $t \in[0, T)$ is such that $F^{\prime}(t)>0$, then (5.12) will be valid, if for such values of $t \in[0, T)$

$$
H(t)+\|u(t)\|_{L^{p+1}(0,1)}^{p+1}+\left\|u^{\prime}(t)\right\|_{L^{2}(0,1)}^{2} \geq C\left[H(t)^{1-\alpha}+\epsilon F^{\prime}(t)\right]^{\frac{1}{1-\alpha}} .
$$

We now prove (5.14). So, assume that $F^{\prime}(t)>0$, and let $\theta=\frac{1}{1-\alpha}$. Since $1<\theta<2$, then by convexity

$$
\left[H(t)^{1-\alpha}+\epsilon F^{\prime}(t)\right]^{\theta} \leq C\left[H(t)+F^{\prime}(t)^{\theta}\right] .
$$


Moreover,

$$
\begin{aligned}
F^{\prime}(t)^{\theta} & =\left(2 \int_{0}^{1} u(t) u^{\prime}(t) d x\right)^{\theta} \\
& \leq C\left(\|u(t)\|_{L^{2}(0,1)}\left\|u^{\prime}(t)\right\|_{L^{2}(0,1)}\right)^{\theta} \\
& \leq C\|u(t)\|_{L^{p+1}(0,1)}^{\theta}\left\|u^{\prime}(t)\right\|_{L^{2}(0,1)}^{\theta} .
\end{aligned}
$$

Since $\frac{2}{\theta}=2(1-\alpha)>1$, by Young's inequality we obtain

$$
F^{\prime}(t)^{\theta} \leq C\left(\left\|u^{\prime}(t)\right\|_{L^{2}(0,1)}^{2}+\|u(t)\|_{L^{p+1}(0,1)}^{\frac{2 \theta}{2-\theta}}\right) .
$$

Now we remark that $\|u(t)\|_{L^{p+1}(0,1)}^{p+1}>(p+1) H(0)>0$. Furthermore, since $\alpha$ was chosen so that $\alpha \leq \frac{p-1}{2(p+1)}$, then $\frac{2 \theta}{2-\theta} \leq p+1$. Therefore, there exists a constant $C_{1}>0$ such that $\|u(t)\|_{L^{p+1}(0,1)}^{\frac{2 \theta}{2-\theta}} \leq C_{1}\|u(t)\|_{L^{p+1}(0,1)}^{p+1}$. Thus, it follows from (5.17) that

$$
F^{\prime}(t)^{\frac{1}{1-\alpha}} \leq C_{2}\left(\left\|u^{\prime}(t)\right\|_{L^{2}(0,1)}^{2}+\|u(t)\|_{L^{p+1}(0,1)}^{p+1}\right),
$$

for some positive constant $C_{2}$.

Therefore, (5.14) is valid. Consequently (5.12) holds, and therefore $y(t)=$ $H(t)^{1-\alpha}+\epsilon F^{\prime}(t)$ blows up in finite time.

\section{REFERENCES}

1. R.A. Adams, Sobolev Spaces, Academic Press, New York 1975. MR 56:9247

2. K. Agre and M.A. Rammaha, Global solutions to boundary value problems for a nonlinear wave equation in high space dimensions, Differential and Integral Equations, 14 (2001), 13151331.

3. Dang Dinh Ang and A. Pham Ngoc Dinh, Mixed problem for some semilinear wave equation with a nonhomogeneous condition, Nonlinear Analysis. Theory, Methods and Applications, 12 (1988), 581-592. MR 89h:35207

4. J. Ball, Remarks on blow-up and nonexistence theorems for nonlinear evolution equations, Quart. J. Math. Oxford (2) 28 (1977), 473-486. MR 57:13150

5. V. Georgiev and G. Todorova, Existence of a solution of the wave equation with nonlinear damping and source terms, J. Diff. Equations 109 (1994), 295-308. MR 95b:35141

6. R.T. Glassey, Blow-up theorems for nonlinear wave equations, Math. Z. 132 (1973), 183-203. MR 49:5549

7. J. Greenberg, R. MacCamy and V. Mizel, On the existence, uniqueness and stability of solutions of the equation $\sigma^{\prime}\left(u_{x}\right) u_{x} x+\lambda_{x t x}=\rho_{0} u_{t t}$, J. Math. Mech. 17 (1968), 707-728. MR 37:023

8. P. Grisvard, Caractérisation de quelques espaces d' interpolation, Arch. Rat. Mech. Anal. 25 (1967), 40-63. MR 35:4718

9. P. Grisvard, Équations différentielles abstraites, Ann. Sci. École Norm. Sup. 2(4) (1969), 311-395. MR 42:5101

10. K. Jörgens, Das Anfangswertproblem im Grossen für eine Klasse nichtlinearer Wellengleichungen, Math. Z. 77 (1961), 295-308. MR 24:A323

11. K. Kawarada, On solutions of nonlinear wave equations, J. Phys. Soc. Japan 31 (1971), 280282. MR 47:5451

12. I. Lasiecka, J.L. Lions and R. Triggiani, Nonhomogeneous boundary value problem for second order hyperbolic operators, J. Math. Pures et Appl. 65 (1986), 149-192. MR 88c:35092

13. I. Lasiecka and R. Triggiani, A cosine operator approach to modelling $L_{2}\left(0, T ; L_{2}(\Gamma)\right)$ boundary input hyperbolic equations, Appl. Math. Optim. 7 (1981), 35-83. MR 82b:35097 
14. I. Lasiecka and R. Triggiani, Regularity theory of hyperbolic equations with non-homogeneous Neumann boundary conditions. II. General boundary data, J. Diff. Equations 94 (1991), 112164. MR 93c:35016

15. H.A. Levine, Instability and nonexistence of global solutions of nonlinear wave equations of the form $P u_{t t}=A u+\mathcal{F}(u)$, Trans. Amer. Math. Soc. 192 (1974), 1-21. MR 49:9436

16. H.A. Levine and J. Serrin, Global nonexistence theorems for quasilinear evolution equations with dissipation, Arch. Rat. Mech. Anal. 137 (1997), 341-361. MR 99b:34110

17. H.A. Levine, S.R. Park, and J.M. Serrin, Global existence and nonexistence theorems for quasilinear evolution equations of formally parabolic type, J. Diff. Equations, 142 (1998), 212-229. MR 99b:35089

18. H.A. Levine, S.R. Park, and J.M. Serrin, Global existence and global nonexistence of solutions of the Cauchy problem for a nonlinearly damped wave equation, J. Math. Anal. Appl., 228 (1998), 181-205. MR 99k:35124

19. J.L. Lions and E. Magenes, Non-Homogeneous Boundary Value Problems and Applications I, II, Springer-Verlag, New York-Heidelberg-Berlin, 1972. MR 50:2670 MR 50:2671

20. J.L. Lions and W.A. Strauss, Some non-linear evolution equations, Bull. Soc. Math. France, 93 (1965), 43-96. MR 33:7663

21. L.E. Payne and D. Sattinger, Saddle points and instability of nonlinear hyperbolic equations, Israel Math. J. 22 (1981), 273-303. MR 53:6112

22. R. Seeley, Interpolation in $L^{P}$ with boundary conditions, Stud. Math. XLIV (1972), 47-60. MR 47:3981

23. I. E. Segal, Non-linear semigroups, Annals of Math. 78 (1963), 339-364. MR 27:2879

24. R. Temam, Navier-Stokes Equations, Theory and Numerical Analysis, North-Holland, 1984. MR 86m:76003

25. H. Tsutsumi, On solutions of semilinear differential equations in a Hilbert space, Math. Japonica 17 (1972), 173-193. MR 50:7723

26. G.F. Webb, Existence and asymptotic behavior for a strongly damped nonlinear wave equation, Can. J. Math. 32 (1980), 631-643. MR 81i:35116

Department of Mathematics and Statistics, University of Nebraska-Lincoln, LinCOLN, NeBraska 68588-0323

E-mail address: rammaha@math.unl.edu

7210 C Eden Brook Drive, \#204, Columbia, Maryland 21046

E-mail address: tastrei@yahoo.com 